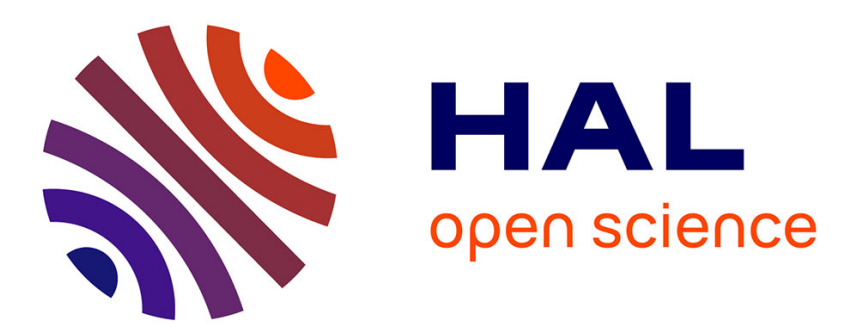

\title{
Modeling population patterns of chemotactic bacteria in homogeneous porous media
}

Florian Centler, Ingo Fetzer, Martin Thullner

\section{To cite this version:}

Florian Centler, Ingo Fetzer, Martin Thullner. Modeling population patterns of chemotactic bacteria in homogeneous porous media. Journal of Theoretical Biology, 2011, 287, pp.82. 10.1016/j.jtbi.2011.07.024 . hal-00730281

\section{HAL Id: hal-00730281 \\ https://hal.science/hal-00730281}

Submitted on 9 Sep 2012

HAL is a multi-disciplinary open access archive for the deposit and dissemination of scientific research documents, whether they are published or not. The documents may come from teaching and research institutions in France or abroad, or from public or private research centers.
L'archive ouverte pluridisciplinaire HAL, est destinée au dépôt et à la diffusion de documents scientifiques de niveau recherche, publiés ou non, émanant des établissements d'enseignement et de recherche français ou étrangers, des laboratoires publics ou privés. 


\section{Author's Accepted Manuscript}

Modeling population patterns of chemotactic bacteria in homogeneous porous media

Florian Centler, Ingo Fetzer, Martin Thullner

PII:

S0022-5193(11)00374-2

DOI: doi:10.1016/j.jtbi.2011.07.024

Reference: YJTBI 6565

To appear in: $\quad$ Journal of Theoretical Biology

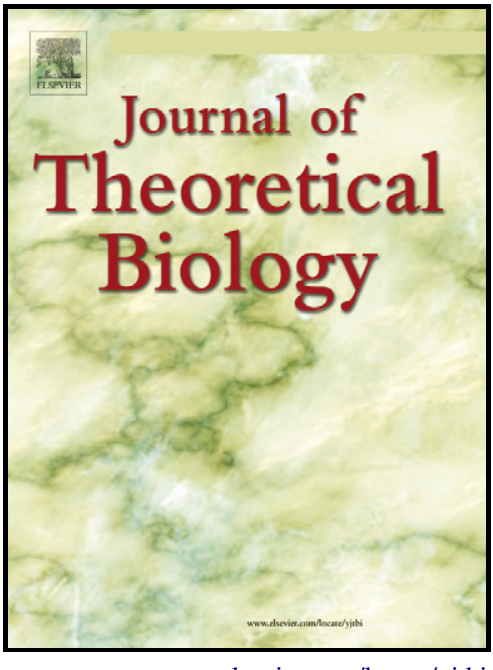

www.elsevier.com/locate/yjtb

Received date: 1 November 2010

Revised date: $\quad 22$ July 2011

Accepted date: 26 July 2011

Cite this article as: Florian Centler, Ingo Fetzer and Martin Thullner, Modeling population patterns of chemotactic bacteria in homogeneous porous media, Journal of Theoretical Biology, doi:10.1016/j.jtbi.2011.07.024

This is a PDF file of an unedited manuscript that has been accepted for publication. As a service to our customers we are providing this early version of the manuscript. The manuscript will undergo copyediting, typesetting, and review of the resulting galley proof before it is published in its final citable form. Please note that during the production process errors may be discovered which could affect the content, and all legal disclaimers that apply to the journal pertain. 


\title{
Modeling Population Patterns of Chemotactic Bacteria in Homogeneous Porous Media
}

\author{
Florian Centler, Ingo Fetzer, Martin Thullner \\ Department of Environmental Microbiology, UFZ - Helmholtz Centre for Environmental \\ Research, Permoserstraße 15, D-04318 Leipzig, Germany
}

Abstract

The spatio-temporal distribution of subsurface microorganisms determines their efficiency in providing essential ecosystem services such as the degradation of organic matter, the remineralization of carbon and nitrogen, or the remediation of anthropogenic contaminants. Populations of motile, chemotactic bacteria have been shown to be capable of pattern formation even in the absence of environmental heterogeneities. Focusing on the water saturated domain of the subsurface (e.g., aquatic sediments, porous aquifers), we analyze this innate capability of bacterial populations in an idealized model of a homogeneous, saturated porous medium. Considering a linear array of connected, identical microhabitats populated by motile, chemotactic bacterial cells, we identify prerequisites for pattern formation, analyze types of patterns, and assess their impact on substrate utilization. In our model, substrate supplied to the microhabitats facilitates bacterial growth, and microbial cells can migrate between neighboring microhabitats due to i) random motility, ii) chemotaxis towards substrate, and iii) self-attraction. A precondition for inhomogeneous population patterns is analytically derived, stating that patterns are possible if the self-attraction exceeds a threshold defined

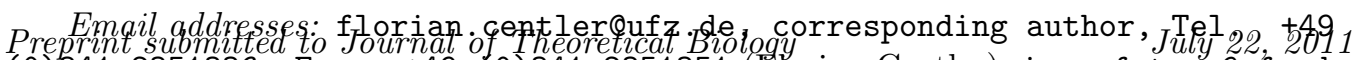

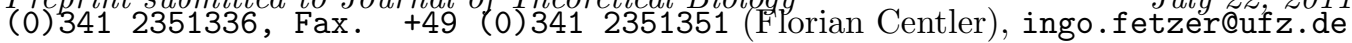
(Ingo Fetzer), martin.thullner@ufz.de (Martin Thullner) 
by the random motility and the steady state population density in the microhabitats. An individual-based implementation of the model shows that static and dynamic population patterns can unfold. Degradation efficiency is highest for homogeneous bacterial distributions and decreases as pattern formation commences. If during biostimulation efforts the carrying capacity of the microhabitats is succesively increased, simulation results show that degradation efficiency can unexpectedly decrease when the pattern formation threshold is crossed.

Key words: pattern formation, ecosystem services, biodegradation, chemotaxis, individual-based modeling

\section{Introduction}

Microbes inhabiting the subsurface domain drive many element cycles which are essential for natural ecosystems and highly desirable from an anthropic viewpoint. They are the main contributors to organic matter breakdown, cycling of carbon and nitrogen, and degradation of organic contaminants in the subsurface. The high spatial and temporal variability of the subsurface domain poses a constant challenge to any microbial life present in this habitat. As a consequence, the organization of subsurface microbial life is complex. The spatial distribution of subsurface bacteria was shown to be highly variable down to the micro-scale, suggesting that bacterial life is inhomogeneously distributed, forming microbial 'hot spots' as local growth conditions vary significantly (Bundt et al., 2001; Nunan et al., 2003). This has profound implications for microbially mediated processes. Taking the mircrobial degradation of contaminants as an example, only the fraction of 
the contaminant can be degraded that comes into close physical contact with the degrading bacteria. Accordingly, bacterial distribution affects contaminant bioavailability and hence degradation efficiency (Harms and Bosma, 1997; Dechesne et al., 2010). In the saturated zone of the subsurface, where the pore space is completely filled with water, as for example in aquatic sediments and aquifers, degradation can be enhanced if bacteria are able to detect contaminant concentration gradients and migrate towards regions of higher concentrations. This process, called chemotaxis, has indeed been shown to be beneficial in the context of bioremediation, where organic contaminants serve as substrate for bacterial growth (Marx and Aitken, 2000; Parales et al., 2000; Ford and Harvey, 2007; Wang et al., 2008). Bacteria in soil often form microcolonies (Chenu et al., 2001; Dechesne et al., 2007). These can originate from a single individual, or be dynamically formed by active aggregation. Such aggregation can be induced by bacterial self-attraction mediated by the excretion of chemoattractants by the cells themselves (Mittal et al., 2003; Park et al., 2003). The amino acids aspartate and glycine have been shown to act as such chemoattractants (Budrene and Berg, 1995; Salman et al., 2006). High cell densities were shown to enhance resistance to environmental stress, for example antibiotics (Butler et al., 2010), promote gene transfer, and allow for efficient extracellular digestion. Furthermore, bacterial aggregation facilitates quorum sensing induced coordinated behavior such as biofilm formation. The microenvironment engineered by bacterial cells in aggregates provides a buffer against environmental fluctuations, enhancing survival. Bacterial self-attraction has extensively been studied for its pattern formation capacity in experiments (see e.g., Budrene and Berg, 1991, 
1995; Ben-Jacob, 2003) and by mathematical modelling (see e.g., Keller and Segel, 1970; Rosen, 1983; Woodward et al., 1995; Tyson et al., 1999; Painter and Hillen, 2002). In these studies, however, usually only one chemotaxis process is considered, with Saragosti et al. (2010) presenting a recent exception focusing on traveling pulses of bacteria. Even in homogeneous environments where local growth conditions do not vary, bacterial distributions can be inhomogeneous and dynamic. In an experimental setup, Keymer et al. (2006) studied a population of Escherichia coli cells colonizing a microfabricated linear array of connected microhabitats. Rich metapopulation dynamics were observed even if all microhabitats were equal in all parameters including substrate supply. The experimental setup resembles a saturated porous medium as found in the natural subsurface, where microbial life is concentrated in microhabitats which are connected to each other by micropores allowing for diffusive substrate exchange and bacterial motion (Grundmann et al., 2001). Inspired by these results, we consider a similar setup in this theoretical study. Omiting and simplifying many aspects of natural saturated porous media as microbial habitats including advection and spatial complexity, we define a homogeneous environment in our model with identical microhabitats and evenly distributed substrate supply. Under such conditions, any observed patterns can be directly attributed to the system's innate pattern formation ability. While following the experimental setup of Keymer et al. (2006) closely in the formulation of our model, the aim of this work is not to reproduce experimental results. Instead, we focus on first, identifying the determinants leading to spatial distribution patterns in our model system as a substitute for a saturated porous medium, and second, on the impact on degradation efficiency 
as a desired ecosystem service. While most modeling studies take only one chemotaxis process into account, we consider two competing chemotactic processes. Chemotaxis towards substrate leads to bacterial dispersal and enhanced degradation efficiency in a system with homogeneously distributed substrate supply, whereas self-attraction leads to bacterial aggregation. Note that survival advantages of bacterial aggregates will not be relfected in the model.

\section{Model}

Following the experimental setup used by Keymer et al. (2006), we model a linear array of microhabitats resembling a saturated porous medium. A single bacterial species populates water filled microhabitats that are connected to the neighbouring microhabitats by channels, enabling bacterial cells to swim from one microhabitat to the next. Our model consists of two parts. The local model describes growth dynamics within microhabitats and the spatial model describes exchange processes between neighboring habitats (Figure 1).

Local Model. For modeling bacterial growth dynamics, we employ the model already suggested by Keymer et al. (2006). Each microhabitat is treated as a homogeneous, well-stirred reactor. Its current state at time $t$ is characterized by the number of bacterial cells occupying it $p(t) \in[0, K]$ and substrate concentration $c(t)$. Assuming an upper limit $c_{\max }$ for substrate concentration, substrate is considered in the model as a relative, unitless index $s(t):=$ $c(t) / c_{\max } \in[0,1]$. Bacterial population dynamics is assumed to follow logistic growth with the logistic term representing space limitation and an additional dependency on substrate concentration (Keymer et al., 2006): 


$$
\frac{d p}{d t}=r(s) \cdot p \cdot\left(1-\frac{p}{K}\right),
$$

with carrying capacity $K$ of a microhabitat and per capita growth rate $r(s)$ for very small populations as defined by

$$
r(s)=\mu s-d,
$$

with parameter $\mu$ describing bacterial growth under maximal substrate concentration $(s=1)$ and bacterial death controlled by parameter $d$. Substrate is supplied to the microhabitat by a generic capacitive process with rate parameter $\lambda$ and consumed during bacterial growth in the microhabitat. Substrate dynamics is hence described by:

$$
\frac{d s}{d t}=\lambda(1-s)-\varepsilon \mu s \frac{p}{K}
$$

with bacterial conversion efficiency $\varepsilon$. Note that the population density $p$ is expressed in cells per habitat. Hence, the consumption term must be scaled to the size of the microhabitat. Assuming that the carrying capacity $K$ scales linearly with microhabitat size, we use it as a substitute for microhabitat size. This ensures that the same number of cells leads to the consumption of the same amount of substrate molecules, independent of microhabitat size. The full dynamics within one habitat is then described by:

$$
\begin{array}{ll}
\frac{d p}{d t}=(\mu s-d) \cdot p \cdot\left(1-\frac{p}{K}\right) & =: g(p, s) \\
\frac{d s}{d t}=\lambda(1-s)-\varepsilon \mu s \frac{p}{K} & =: h(p, s) .
\end{array}
$$


Spatial Model. Bacterial migration between neighbouring microhabitats is governed by three aspects. First, motile cells perform a random-walk movement as they swim through the fluid medium by rotating their flagella. This process can be described as Fickian diffusion (Berg, 1983; Berg and Turner, 1990). The diffusive flux is $J_{D}=-D_{p} \cdot \nabla p$, with diffusion coefficient $D_{p}$. Second, cells respond to gradients in substrate concentration by directing their movement towards locations of higher concentration. Usually, the chemotactic flux is described as $J_{C}=\chi(c, p) \cdot \nabla c$, with chemotaxis response function $\chi(c, p)$ and chemoattractant concentration $c$. For simplicity, we choose a chemotaxis response function which is linear in population density and independent from chemoattractant concentration. Choosing a constant sensitivity for chemotaxis to substrate $\chi_{s}$, our chemotactic flux towards substrate becomes $J_{s}=\chi_{s} \cdot p \cdot \nabla s$. For the third aspect of cell motility, we assume that bacterial cells excrete molecules that also act as chemoattractants. Instead of considering the chemoattractant explicitely in the model, we assume that its concentrations is proportional to bacterial cell density. Hence, the bacterial flux due to self-attraction can be defined in analogy to the substrate chemotaxis as: $J_{p}=\chi_{p} \cdot p \cdot \nabla p$, with sensitivity $\chi_{p}$. We assume a strictly additive integration of both chemotactic processes (Saragosti et al., 2010). The substrate is assumed to diffuse with diffusion coefficient $D_{s}$. Bringing it all together, the spatial processes are described by two partial differential equations: 


$$
\begin{aligned}
& \frac{\partial p}{\partial t}=D_{p} \cdot \nabla^{2} p-\chi_{s} \cdot \nabla \cdot(p \nabla s)-\chi_{p} \cdot \nabla \cdot(p \nabla p) \\
& \frac{\partial s}{\partial t}=D_{s} \cdot \nabla^{2} s .
\end{aligned}
$$

158

159

Note that the parameters $D_{p}, D_{s}, \chi_{p}$, and $\chi_{s}$ are effective parameters that implicitely account for any flux restrictions imposed by the specific geometry of the corridors that connect neighboring microhabitats.

Full Model. Combining the spatial model (Equations 6 and 7) with the local growth model (Equations 4 and 5) gives the full model:

$$
\begin{array}{rlrl}
\frac{\partial p}{\partial t}= & D_{p} \cdot \nabla^{2} p-\chi_{s} \cdot \nabla \cdot(p \nabla s)-\chi_{p} \cdot \nabla \cdot(p \nabla p) & \\
& +g(p, s) & =: u(p, s) \\
\frac{\partial s}{\partial t}= & D_{s} \cdot \nabla^{2} s & & \\
& +h(p, s) & =: v(p, s) .
\end{array}
$$

\section{Model Analysis}

\subsection{Local Model}

The local model (Equations 4 and 5) has three steady states with $g\left(p^{*}, s^{*}\right)=$ $0, h\left(p^{*}, s^{*}\right)=0$ :

$$
\begin{aligned}
& \left(p_{1}^{*}, s_{1}^{*}\right)=(0,1) \\
& \left(p_{2}^{*}, s_{2}^{*}\right)=(K, \lambda /(\lambda+\varepsilon \mu)) \\
& \left(p_{3}^{*}, s_{3}^{*}\right)=(K \lambda / \varepsilon(1 / d-1 / \mu), d / \mu) .
\end{aligned}
$$


The first steady state refers to the trivial case of bacterial extinction. In the second steady state, the bacterial density is fixed to $K$, representing carrying capacity limited growth. An increase in substrate supply $\lambda$ does not lead to an increase in population density, but only to an increase in substrate concentration. In the third state however, an increase in substrate supply translates into a larger population density, while substrate concentration is fixed. This state refers to growth limited by substrate supply. To elucidate the stability of these steady states, we perform a linear stability analysis. The system is linearized around the steady state and the evolution of a small perturbation is studied. If all eigenvalues of the system's Jacobian matrix are negative, perturbations vanish with time. We find that asymptotic stability is given under following conditions for the three steady states:

$$
\begin{aligned}
& \left(p_{1}^{*}, s_{1}^{*}\right): d>\mu \\
& \left(p_{2}^{*}, s_{2}^{*}\right): d<\mu \lambda /(\lambda+\varepsilon \mu) \\
& \left(p_{3}^{*}, s_{3}^{*}\right): d<\mu, \varepsilon>\lambda(1 / d-1 / \mu) .
\end{aligned}
$$

The extinction case $\left(p_{1}^{*}, s_{1}^{*}\right)$ is only asymptotically stable if the parameter controlling death $d$ exceeds the parameter controlling growth $\mu$. The opposite must be true for the other two steady states to become stable. For the second steady state $\left(p_{2}^{*}, s_{2}^{*}\right)$, parameter $d$ is even more limited, as $\lambda /(\lambda+\varepsilon \mu)<1$. This inequality can be rewritten as $d<\mu s_{2}^{*}$. For the substrate limited steady state $\left(p_{3}^{*}, s_{3}^{*}\right)$, the substrate conversion efficiency must exceed a threshold defined by the parameters for substrate supply, bacterial growth and death. This inequality can be rewritten to $p_{3}^{*}<K$. While the eigenvalues for the 
first two steady states are never complex, the eigenvalues for the third steady state can be complex for certain parameter settings ${ }^{1}$, indicating oscillatory behavior. For a specific example, parameter regions with signs of eigenvalues are shown in Figure 2 for steady states two and three. The bifurcation diagram in Figure 3 illustrates how the system undergoes a transcritical bifurcation. For large values of $\lambda$, steady state two is asymptotically stable and steady state three unstable. Decreasing $\lambda$, the stability properties are switched at the bifurcation point, where saddle nodes collide with stable nodes. Decreasing $\lambda$ further, a second bifurcation occurs for steady state three where the stable node becomes a stable spiral.

\subsection{Full Model}

Mass exchange between microhabitats in the full model is driven by spatial gradients. If all microhabitats have identical population densities and substrate concentrations, spatial gradients vanish and exchange processes will not change the system's state. Hence, if the spatial model is homogeneously initialized with population densities and substrate concentrations of a steady state of the local model, also the full model is at steady state. To assess the stability of the steady state in the full model, a second linear stability analysis needs to be performed. Again, we have to analyze whether a perturbation of the spatial system in steady state is amplified or decays over time. In cases where perturbations do not fade, spatial patterns can emerge. We restrict our analysis to a one-dimensional domain of length $L$ with spatial coordinate $x$. We follow the evolution of small perturbations around any spatially

\footnotetext{
${ }^{1} \lambda<4 d^{2}(\mu-d) / \mu^{2}, \varepsilon>4 \lambda d(\mu-d)^{2} /\left(\mu\left(4 d^{2}(\mu-d)-\lambda \mu^{2}\right)\right)$
} 
210 211 212

homogeneous steady state $(p(x), s(x))=\left(p^{*}, s^{*}\right)$, which is assumed to be asymptotically stable in the local model. We define the perturbation as:

$$
d(x, t):=\left(\begin{array}{c}
p(x, t)-p^{*} \\
s(x, t)-s^{*}
\end{array}\right) .
$$

${ }_{212}$ The time evolution of the perturbation $d$ is given by

$$
\begin{aligned}
\frac{\partial d}{\partial t} & =A d \\
& =\left(\begin{array}{cc}
u_{p} & u_{s} \\
v_{p} & v_{s}
\end{array}\right)_{p^{*}, s^{*}} d \\
& =\left(\begin{array}{rr}
D_{p} \nabla^{2}-\chi_{p} p^{*} \nabla^{2}+g_{p}^{*} & -\chi_{s} p^{*} \nabla^{2}+g_{s}^{*} \\
h_{p}^{*} & D_{s} \nabla^{2}+h_{s}^{*}
\end{array}\right) d,
\end{aligned}
$$

with $u_{p}$ denoting the partial derivative of $u$ with respect to $p$, and $u_{s}, v_{p}, v_{s}$ accordingly, and writing $g_{p}^{*}$ short for $g_{p}\left(p^{*}, s^{*}\right)$, and $g_{s}^{*}, h_{p}^{*}, h_{s}^{*}$ accordingly. With solutions of the form $d \sim \cos (k x) e^{\lambda t}$, with wavenumber $k=n \pi / L, n$ a whole number, the sign of $\lambda$ decides on the fate of the perturbation. From the solution follows: $\nabla^{2} d=-k^{2} d$. Setting this into Equation 17, we can compute $\lambda$ as the eigenvalues of $A$. They are determined by:

$$
\left|\begin{array}{cc}
-k^{2} D_{p}+k^{2} \chi_{p} p^{*}+g_{p}^{*}-\lambda & k^{2} \chi_{s} p^{*}+g_{s}^{*} \\
h_{p}^{*} & -k^{2} D_{s}+h_{s}^{*}-\lambda
\end{array}\right|=0 .
$$

This leads to:

$$
\lambda^{2}+a\left(k^{2}\right) \lambda+b\left(k^{2}\right)=0, \text { with }
$$




$$
\begin{aligned}
a\left(k^{2}\right)= & \left(D_{s}+D_{p}-\chi_{p} p^{*}\right) k^{2}-\left(g_{p}^{*}+h_{s}^{*}\right) \\
b\left(k^{2}\right)= & \left(D_{p}-\chi_{p} p^{*}\right) D_{s} k^{4}-\left(D_{p} h_{s}^{*}+\chi_{s} p^{*} h_{p}^{*}-\chi_{p} p^{*} h_{s}^{*}+D_{s} g_{p}^{*}\right) k^{2}+ \\
& g_{p}^{*} h_{s}^{*}-g_{s}^{*} h_{p}^{*} .
\end{aligned}
$$

224

Spatial patterns can form if $\lambda$ has positive real components, as perturbations do not fade in this case. This is true if $a\left(k^{2}\right)<0$ or $b\left(k^{2}\right)<0$. Assuming that the steady state $\left(p^{*}, s^{*}\right)$ is asymptotically stable in the local model, it follows that both of its eigenvalues $\lambda_{l}^{1,2}$ are negative in the local model. From $\left|\begin{array}{cc}g_{p}^{*}-\lambda_{l} & g_{s}^{*} \\ h_{p}^{*} & h_{s}^{*}-\lambda_{l}\end{array}\right|=0$, with $\lambda_{l}^{1,2}<0$ follows: $g_{p}^{*}+h_{s}^{*}<0, g_{p}^{*} h_{s}^{*}-g_{s}^{*} h_{p}^{*}>0$. Using this we find that $a\left(k^{2}\right)<0$ for $k>k_{\text {crit }}$, if $D_{s}+D_{p}-\chi_{p} p^{*} \leq 0$. Furthermore, $b\left(k^{2}\right)<0$ is guaranteed for $k>k_{\text {crit }}^{\prime}$, if $D_{p}-\chi_{p} p^{*}<0$. And finally for $D_{p}-\chi_{p} p^{*}>0, b\left(k^{2}\right)$ might still become negative for $k_{c r i t, l}<k<$ $k_{c r i t, h}$. Concluding, spatial patterns are in principle possible for $D_{p}-\chi_{p} p^{*}>$ 0 , albeit under a limited choice of wave numbers $k$. This limitation is relaxed for

$$
D_{p}-\chi_{p} p^{*}<0
$$

Under this condition, spatial patterns can be expected.

\section{Individual-Based Model Implementation}

Microcolonies consisting of very few individual cells have been reported in the subsurface (Chenu et al., 2001; Grundmann, 2004). Since differential equation based modeling is not applicable to populations of few cells and 
additionally, blow up is a problem in chemotaxis modeling using partial differential equations (see e.g., Perthame, 2007), we follow an individual-based approach. This approach has been successfully applied in microbial ecology, for example in the context of biofilms (see e.g., Kreft et al., 2001; Mabrouk et al., 2010). The differential equation based full model focusing on the population level is converted into a stochastic model implementation focusing on the single cell level for computational simulations. The simulation loop consists of two steps: First, the growth dynamics is simulated for each microhabitat in the array. In the second step, bacterial migration between neighboring microhabitats, and substrate diffusion is simulated. Within the individual-based simulation, each bacterial cell is considered separately. Bacterial processes considered in the model are not assumed to depend on a specific state of the cells such as age or size, so that cells can be modeled as stateless entities. For the local model, we need to derive probabilities for the stochastically independent events of cell division $P_{\mu}$ and cell death $P_{d}$ during each step of the simulation of length $\Delta t$. Rewriting Equation 4 as

$$
\frac{d p}{d t}=p \cdot\left(\mu s-(\mu s-d) \frac{p}{K}\right)-p \cdot d
$$

allows us to assign the probabilities as:

$$
\begin{aligned}
P_{\mu} & :=\left(\mu s-(\mu s-d) \frac{p}{K}\right) \cdot \Delta t \\
P_{d} & :=d \cdot \Delta t .
\end{aligned}
$$

In the first step of the simulation loop, each bacterial cell has the chance to divide and die, in accordance with these probabilities. For each bacterial 
cell, two uniformly distributed random numbers $r_{\mu}$ and $r_{d}$ are drawn over the intervall $[0,1]$. The cell divides in case of $r_{\mu}<P_{\mu}$ and (additionally) dies in case of $r_{d}<P_{d}$. The time step size has to be small enough to ensure $P_{\mu}$ and $P_{d} \ll 1$. To derive probabilities for the migration of bacteria, we apply the standard central difference scheme to Equation 6. The resulting equation can be arranged to:

$$
p_{i}^{t+\Delta t}=P_{0} \cdot p_{i}^{t}+P_{1} \cdot p_{i+1}^{t}+P_{2} \cdot p_{i-1}^{t},
$$

with

$$
\begin{aligned}
P_{0}= & 1-\frac{2 D_{p} \Delta t}{(\Delta x)^{2}}-\frac{\chi_{s} \Delta t}{(\Delta x)^{2}}\left(s_{i+1}-2 s_{i}+s_{i-1}\right)- \\
& \frac{\chi_{p} \Delta t}{(\Delta x)^{2}}\left(p_{i+1}-2 p_{i}+p_{i-1}\right) \\
P_{1}= & \frac{D_{p} \Delta t}{(\Delta x)^{2}}-\frac{\chi_{s} \Delta t}{4(\Delta x)^{2}}\left(s_{i+1}-s_{i-1}\right)-\frac{\chi_{p} \Delta t}{4(\Delta x)^{2}}\left(p_{i+1}-p_{i-1}\right) \\
P_{2}= & \frac{D_{p} \Delta t}{(\Delta x)^{2}}-\frac{\chi_{s} \Delta t}{4(\Delta x)^{2}}\left(s_{i-1}-s_{i+1}\right)-\frac{\chi_{p} \Delta t}{4(\Delta x)^{2}}\left(p_{i-1}-p_{i+1}\right),
\end{aligned}
$$

where $p_{i}^{t}$ describes the number of bacterial cells in microhabitat $i$ at time $t$, $s_{i}$ describes the substrate concentration in habitat $i$ at time $t$ and $\Delta x$ describes the distance between neighboring microhabitats. We follow Schofield et al. (2002) who take the coefficients $P_{0}, P_{1}$, and $P_{2}$ to be proportional to the probability for a bacterial cell to remain at its current microhabitat $\left(P_{0}\right)$, move to the left microhabitat $\left(P_{1}\right)$, and move to the right microhabitat $\left(P_{2}\right)$. For simulating cell migration in each time step, a random number $q$ is drawn for each bacterial cell in the array that is uniformly distributed over the interval $\left[0, \sum_{k} P_{k}\right]$. For $q \in\left[0, P_{0}[\right.$, the cell remains in its current microhabitat, 
for $q \in\left[P_{0}, P_{1}[\right.$, the cell migrates to the left neighboring microhabitat and for $q \in\left[P_{1}, P_{2}\right]$ it migrates to its right neighbor microhabitat. In order to avoid boundary effects at the edge of the microhabitat array, we employ periodic boundary conditions in which the array is wrapped around forming a ring, so that the rightmost habitat has the leftmost habitat as its right neighbor. If, after all migratory events have been performed, a habitat contains more cells than its carrying capacity $K$, cells are randomly moved from the overcrowded habitat to its neighboring habitats until all habitats contain at most $K$ cells. For the substrate diffusion, the standard central difference scheme is directly applied to Equation 7.

Parameter Settings. We simulate an array of 80 microhabitats. All parameter values are summarized in Table 1. The parameters for the local model are taken from Keymer et al. (2006) who fitted them to experimental data for a single habitat. With these parameter settings, only the third steady state, refering to substrate limited growth, is asymptotically stable with $\left(p_{3}^{*}, s_{3}^{*}\right)=(2747$ cells, 0.394$)$. This steady state is a stable spiral. For the diffusion coefficient for substrate, we choose a value which is typical for small molecules (Berg, 1983), for example toluene or benzene. For the diffusion coefficient of the bacteria and their chemotactic sensitivity towards the substrate, we use experimentally derived values from Berg and Turner (1990). They set up capillary tubes with a diameter of $10 \mu \mathrm{m}$ to simulate a porous medium consisting of straight pores. We vary the sensitivity for self-attraction over one order of magnitude. For the upper limit, we assume that the self-attraction migration flux does not exceed the flux due to chemotaxis towards substrate, as the response to substrate is supposed to be the 
most pronounced due to its direct evolutionary advantage. Assuming the maximum gradients for bacterial cells and substrate concentration, the contributions of both chemotactic processes to the migration probability become equal if setting $\chi_{p}^{\max }=\chi_{s} / K$. This defines the upper limit of the chemotactic sensitivity for self-attraction. In all simulation runs of the full model, each microhabitat in the array is initialized at steady state three of the local model with $\left(\left(p^{t_{0}}, s^{t_{0}}\right)=(2747,0.394)\right)$.

\section{Simulation Results}

\subsection{Bacterial Growth in the Local Model}

First, only growth within a single habitat is considered and the deterministic solution of the local model (Equations 4 and 5) is compared to ten independent runs of the individual-based model. The system is initialized either with ten bacterial cells and maximal substrate concentration $\left(\left(p^{t_{0}}, s^{t_{0}}\right)=(10,1.0)\right)$ or at steady state three $\left(\left(p^{t_{0}}, s^{t_{0}}\right)=(2747,0.394)\right)$. In the former case (Figure 4, left), a damped oscillation around the steady state is visible after a first exponential growth phase, in accordance with steady state three being a stable spiral. The stochastic runs generally follow the deterministic dynamics with stochastic fluctuations leading to a time offset and varying amplitudes, scattered around the deterministic amplitude. An erratic oscillation of the stochastic system around the deterministic solution is visible when the system is initialized at steady state three (Figure 4, right). 


\subsection{Homogeneous Bacterial Distributions in the Full Model}

According to Equation 22, homogeneous bacterial distributions can be expected for the chosen parameter set if the chemotactic sensitivity towards the chemoattractant $\chi_{p}$ is below the critical value of $\chi_{p}^{*}=1.89 \times 10^{-9} \mathrm{~cm}^{2} / \mathrm{s}$. In this case, the whole microhabitat array is homogeneously populated over time except for stochastic fluctuations (Figure 5, left). If considering the evolution of mean values over all microhabitats, an oscillation in population density and substrate concentration close to the local steady state three becomes apparent (Figure 5, right). A high population density is followed by a decrease in substrate concentration, which in turn leads to a decreasing population. This allows the microhabitat to recover. With the ensuing increase in population density, the circle is closed. In this case, the mean dynamics of the whole system resembles the local dynamics. The array can be thought of as operating as one large microhabitat.

\subsection{Pattern Formation}

If the chemotactic sensitivity $\chi_{p}$ is set to values above the pattern formation threshold $\chi_{p}^{*}$, inhomogeneous spatial population distributions emerge. In the transition from homogeneous distributions to spatial structures, four types of distinct spatio-temporal distribution patterns can be distinguished (Figure 6). Pattern formation starts shortly before the threshold $\chi_{p}^{*}$ is reached. In the first pattern type, few microhabitats spontaneously become saturated with bacterial cells. Microhabitats in the vicinity of these static hot spots are depleted in bacteria, while distant microhabitats are still homogeneously populated. As $\chi_{p}$ is increased, more and more static hot spots form, until hot spots start to repeatedly form and disintegrate in Pattern Type 2. The 
remaining microhabitats synchronously oscillate in population density. The formation of new hot spots is triggered by peaks in mean population density, whereas disintegration is induced by low mean densities. While some hot spots last for only one oscillation, others prevail over longer time periods. As $\chi_{p}$ is further increased, only few evenly distributed hot spots form in the array in Pattern Type 3 of the transition. They span up to two microhabitats and occasionally shift to neighboring microhabitats. Increasing $\chi_{p}$ further leads to a dense pattern of static hot spots next to almost completely vacated microhabitats in the final Pattern Type 4 of the transition. The four transition pattern types also show distinct dynamics in the mean behavior of the whole array (Figure 7). The trajectory of the mean population density and mean substrate concentration in Pattern Type 1 remains in the vicinity of the local steady state three, albeit shifted to a state of slightly lower density and higher substrate concentration. The dynamic pattern of Pattern Type 2 corresponds to a pronounced oscillation of the mean trajectory. As few moving hot spots form in Pattern Type 3, the mean trajectory settles in an oscillation of small amplitude far away from steady state three after an initial transient phase. For Pattern Type 4, the mean trajectory settles in an oscillation around a state of lower density and higher substrate concentration compared to steady state three, albeit in closer proximity to it than in Pattern Type 3. Here, the amplitude of the oscillation is smaller compared to the oscillation in the spatially homogeneous case (cf. insets in Figure 7 and Figure 5, right). Mean population density per microhabitat steadily decreases during the first three pattern types of the transition. Although density begins to recover in the Pattern Type 4, the level achieved 
under homogeneous colonization is not reached again (Figure 8). Due to fast substrate diffusion, substrate gradients remain small in the microhabitat array and play a minor role in bacterial migration. A decrease by a factor of 50 brings the substrate diffusion coefficient into the range that has been reported for the herbicide atrazine in soil (Ritter et al., 1973). Under these conditions, substrate gradients affect bacterial migration. A typical simulation run with $\chi_{p}>\chi_{p}^{*}$ is shown in Figure 9. At the begining of the simulation, a transient checkerboard pattern unfolds. Substrate is faster consumed in highly populated microhabitats. This makes neighboring microhabitats of lower cell densities more and more attractive, eventually leading to a migration to these microhabitats. Vacated microhabitats recover in substrate concentration due to permanent substrate supply, again becoming attractive for recolonization. After approximately ten minutes, traveling waves emerge that sweep through the array, attracted by fresh microhabitats and leaving substrate depleted microhabitats behind. On collision, migratory waves create temporal hot spots that finally dissolve and release further migration waves. Despite the highly structured spatio-temporal bacterial distribution, the mean behavior of the whole array shows little variability (Figure 9, right). As bacterial activity is concentrated in few microhabitats, an overall state of lower mean population density and higher mean substrate concentration is assumed in comparison to the local steady state three.

\subsection{Effect of Biostimulation on Substrate Utilization}

In order to enhance the remediation of contaminated field sites, biostimulation has been proposed (Wenderoth et al., 2003). For example, additional terminal electron acceptors can be injected into the contaminated subsurface 
to stimulate growth of degrading bacteria. In our model, the carrying capacity $K$ describes the maximum number of cells that can be supported in one microhabitat. This limit depends on microhabitat size, and can additionally also depend on further requirements for bacterial growth, for example terminal electron acceptors such as nitrate or sulfate, which are not explicitely considered in our model. Hence, an addition of terminal electron acceptors translates into an increase of $K$ in our model. Combining the pattern formation condition Equation 22 with the condition for steady state three representing substrate limited growth (Equation 12) leads to:

$$
D_{p}<K \chi_{p} \lambda / \varepsilon \cdot(1 / d-1 / \mu)
$$

as the condition for pattern formation. This formulation makes it evident that an increasing value of $K$ due to biostimulation efforts can lead to the pattern formation condition to become true and hence the emergence of spatially inhomogeneous bacterial population patterns. To evaluate the resulting effect on substrate degradation as an desired ecosystem function, we perform a series of simulation runs with increasing $K$ values and consider the mean consumption rate and mean population density over the whole array and entire simulation time, excluding the initial transient phase (Figure 10). The consumption rate is computed as substrate concentration decrease due to microbial growth (second summand in Equation 5, but without scaling factor $K)$. Chemotactic sensitivity towards chemoattractant is set to $1.3 \times 10^{-9}$ $\mathrm{cm}^{2} / \mathrm{s}$. Under these parameter settings, the pattern formation threshold for the carrying capacity $K$ is $K^{*}=14560$ bacterial cells. The response to biostimulation can be divided into three stages (Figure 10). For low $K$ values, 
the array is homogeneously populated and its dynamics follows the dynamics of the local model. An increase in $K$ leads to a linear increase in population density and consumption rate. As the pattern formation threshold $K^{*}$ is almost reached, the population density and consumption rate decrease, despite the availabilty of better growth conditions due to larger $K$ values. As bacterial activity becomes concentrated in hot spot microhabitats, substrate available in microhabitats of low bacterial density is no longer utilized. Hence, the onset of spatial pattern formation leads to a nonlinear response to biostimulation. The trend is reversed at $K$ values above 16000 cells, where the linear response resumes. As more and more fully saturated microhabitats form, more substrate can be utilized. However, the maximum consumption rate given by steady state three of the local model cannot be reached again once the pattern formation threshold has been crossed.

\section{Discussion}

Studying the dynamics of a single bacterial species colonizing a linear array of connected microhabitats in a simplistic model resembling a saturated porous medium led to three main results. The first result was obtained by an analytical analysis that revealed a critical threshold above which spatially inhomogeneous population patterns are possible. The existence of patterns depends on the relation between bacterial parameters and environmental factors. This becomes apparent if the pattern formation condition (Equation 30) is rewritten as: 


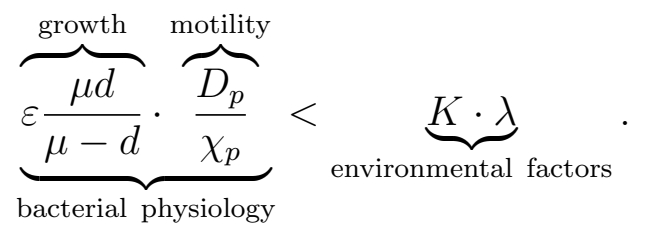

This relation indicates that for fixed environmental factors, faster bacterial growth, a lower random motility, as well as an increased affinity to fellow bacteria will increase the likelihood of inhomogeneous bacterial distributions. The parameter values for bacterial growth and death used in the simulations were taken from experimental data on E. coli cells. The chosen maximal bacterial growth rate of $0.15 \mathrm{~min}^{-1}$ is beyond the capacity of typical soil bacteria. If both growth and death rate are reduced by the same factor to values more realistic for soil bacteria, the growth term in Equation 31 gets reduced by the same factor. This shifts the pattern formation threshold to lower values of $\chi_{p}$, making inhomogeneous distributions more probable. This trend however is counteracted if for the environmental factors a lower carrying capacity or substrate supply rate is chosen, in accordance with substrate usually being scarce in subsurface environments. Bacterial physiology parameters can vary between bacterial species. Hence, under a given environment, one species might tend to form patterns while the other does not. And vice versa, if environmental factors vary over space and time, a species might tend to form patterns only in specific locations and during distinct periods of time. Both the diffusion constant for substrate and the chemotactic sensitivity towards the substrate do not appear in the pattern formation condition. Under the homogeneous environment conditions considered in our model, they hence have no influence on the existence of patterns in the 
model. As the second result of this study, the potential types of spatiotemporal patterns were identified using individual-based simulation runs. It is well known that complex patterns can spontaneously emerge in coupled reaction-diffusion systems even under homogeneous environments (Turing, 1952). Turing patterns of bacteria have already been reported in a sediment modeling study by Baurmann et al. (2004). For parameter settings far away from the pattern formation threshold, static patterns emerged in our model: homogeneous bacterial distributions if parameters were set to values below the threshold, and a pattern of saturated microhabitats next to virtually empty microhabitats if parameter values exceeded the threshold. The whole array operates as one large microhabitat for homogeneous bacterial distributions. For inhomogeneous distributions, the mean behavior of the whole microhabitat array deviates from the local model's dynamics and a state of lower mean population density and higher mean substrate concentration is assumed. Furthermore, the variability of mean bacterial population and substrate concentration over time was reduced in these cases. Hence, under pattern formation, the spatial interactions exerted a stabilizing effect on habitat dynamics. Dynamic patterns were found for parameter values in the vicinity of the pattern formation threshold. In particular, if substrate diffusion was decreased, dynamic checkerboard and wave patterns emerged. Such wave like motions have also been observed in experimental setups (Keymer et al., 2006; Saragosti et al., 2010). As the third result of this study, the onset of pattern formation was found to cause a non-linear response to biostimulation efforts. It has been experimentally shown that aggregated bacterial distribution patterns lead to reduced biodegradation (Dechesne et al., 2010). 
A similar phenomenon was observed in our model. Biodegradation efficiency decreased as the pattern formation threshold was exceeded and the bacterial distribution became inhomogeneous. As bacterial activity becomes concentrated in few hot spots, substrate in microhabitats of low bacterial density is no longer degraded efficiently. This fraction of the substrate is no longer bioavailable due to the absence of bacteria, and the overall degradation performance falls short of the expectation derived from the local dynamics. This is a common observation when applying laboratory results to the field-scale. In our model, pattern formation is the cause leading to reduced bioavailability and hence, reduced overall degradation performance. In a field application, however, the non-linear response to biostimulation can only be observed if the microhabitats are homogeneously colonized in the pristine state. And even then, it could be argued that by further increasing biostimulation after the onset of pattern formation and ancompanying decrease in degradation performance, the linear response will resume. In this study, a simplistic model has been used that omits and simplifies many aspects of the subsurface as a bacterial habitat. For example, a water phase was assumed to be present that connects neighbouring microhabitats, advection has been neglected, and microhabitats were identical, mimicking a homogeneous environment. Furthermore, microhabitats were treated as well-mixed, neglecting any sub-scale spatial features, and only a single bacterial species has been considered in contrast to the tremendous diversity of soil bacteria. These simplifications, however, allowed us to analytically derive a condition for pattern formation, and uncover mechanisms driving spatio-temporal bacterial distribution, that are also plausible driving forces for the dynamics in real soil systems. Bacte- 
rial growth was modeled following a modified logistic growth model suggested by Keymer et al. (2006). Note however, that the analytical derivation of the pattern formation condition did not depend on a particular growth model. The existence of an asymptotically stable steady state was the only assumption regarding the local growth model. Hence, the results are also valid if a different growth model is used, for example if bacterial growth follows Monod-type kinetics. The model can easily be extended to become a more faithful representation of the subsurface environment. Spatial inhomogeneity of the environment can be included by varying carrying capacity and substrate inflow over microhabitats. The influence of heterogeneities imposed by the environment on the innate pattern formation capability of bacterial populations can then be studied. Furthermore, an additional bacterial species can be included. In such a scenario, bacterial interactions and coexistence in spatially structured environments can be assessed. If parameters describing bacterial physiology including chemotactic sensitivities are subjected to random mutations on cell division, the evolution of survival strategies of both species can be analyzed. In the presented work, mathematical and computational modeling was applied as a suitable tool to advance our understanding of the dynamics of microbial processes in the subsurface. While not all parameters of interest are necessarily available at the desired spatio-temporal resolution in experimental setups, computational models are not constrained by such limitations. This makes them well suited to identify key mechanisms behind microbially driven dynamics in the subsurface. 
531

532

\section{Acknowledgments}

This work was supported by the Helmholtz Association Grant VG-NG-338 "GReaT MoDE" and in addition via research topic CITE - Chemicals in the Environment.

\section{References}

Baurmann, M., Ebenhöh, W., Feudel, U., 2004. Turing instabilities and pattern formation in a benthic nutrient-microoganism system. Math. Biosci. Eng. 1, 111-130.

Ben-Jacob, E., 2003. Bacterial self-organization: co-enhancement of complexification and adaptability in a dynamic environment. Philos. Transact. A Math. Phys. Eng. Sci. 361, 1283-1312.

Berg, H.C., 1983. Random Walks in Biology. Princeton University Press, Princeton, NJ.

Berg, H.C., Turner, L., 1990. Chemotaxis of bacteria in glass capillary arrays. Biophys. J. 58, 919-930.

Budrene, E.O., Berg, H.C., 1991. Complex patterns formed by motile cells of Escherichia coli. Nature 349, 630-633.

Budrene, E.O., Berg, H.C., 1995. Dynamics of formation of symmetrical patterns by chemotactic bacteria. Nature 376, 49-53.

Bundt, M., Widmer, F., Pesaro, M., Zeyer, J., Blaser, P., 2001. Preferential flow paths: biological 'hot spots' in soils. Soil. Biol. Biochem. 33, 729-738. 
Butler, M.T., Wang, Q., Harshey, R.M., 2010. Cell density and mobility protect swarming bacteria against antibiotics. Proc. Natl. Acad. Sci. USA 107, 3776-3781.

Chenu, C., Hassink, J., Bloem, J., 2001. Short-term changes in the spatial distribution of microorganisms in soil aggregates as affected by glucose addition. Biol. Fertil. Soils. 34, 349-356.

Dechesne, A., Owsianiak, M., Bazire, A., Grundmann, G.L., Binning, P.J., Smets, B.F., 2010. Biodegradation in a partially saturated sand matrix: compounding effects of water content, bacterial spatial distribution, and motility. Environ. Sci. Technol. 44, 2386-2392.

Dechesne, A., Pallud, C., Grundmann, G.L., 2007. Spatial distribution of bacteria at the microscale in soil, in: Franklin, R.B., Mills, A.L. (Eds.), The Spatial Distribution of Microbes in the Environment. Springer. chapter 4, pp. $87-107$.

Ford, R.M., Harvey, R.W., 2007. Role of chemotaxis in the transport of bacteria through saturated porous media. Adv. Water. Resour. 30, 16081617.

Grundmann, G.L., 2004. Spatial scales of soil bacterial diversity-the size of a clone. FEMS Microbiol. Ecol. 48, 119-127.

Grundmann, G.L., Dechesne, A., Bartoli, F., Flandrois, J.P., Chassé, J.L., Kizungu, R., 2001. Spatial modeling of nitrifier microhabitats in soil. Soil. Sci. Soc. Am. J. 65, 1709-1716. 
Harms, H., Bosma, T.N.P., 1997. Mass transfer limitation of microbial growth and pollutant degradation. J. Ind. Microbiol. Biot. 18, 97-105.

Keller, E.F., Segel, L.A., 1970. Initiation of slime mold aggregation viewed as an instability. J. Theor. Biol. 26, 399-415.

Keymer, J.E., Galajda, P., Muldoon, C., Park, S., Austin, R.H., 2006. Bacterial metapopulations in nanofabricated landscapes. Proc. Natl. Acad. Sci. USA 103, 17290.

Kreft, J.U., Picioreanu, C., Wimpenny, J.W., van Loosdrecht, M.C., 2001. Individual-based modelling of biofilms. Microbiology 147, 2897-2912.

Mabrouk, N., Deffuant, G., Tolker-Nielsen, T., Lobry, C., 2010. Bacteria can form interconnected microcolonies when a self-excreted product reduces their surface motility: evidence from individual-based model simulations. Theory. Biosci. 129, 1-13.

Marx, R.B., Aitken, M.D., 2000. Bacterial chemotaxis enhances naphthalene degradation in a heterogeneous aqueous system. Environ. Sci. Technol. 34, 3379-3383.

Mittal, N., Budrene, E.O., Brenner, M.P., Oudenaarden, A.V., 2003. Motility of Escherichia coli cells in clusters formed by chemotactic aggregation. Proc. Natl. Acad. Sci. USA 100, 13259-13263.

Nunan, N., Wu, K., Young, I.M., Crawford, J.W., Ritz, K., 2003. Spatial distribution of bacterial communities and their relationships with the micro-architecture of soil. FEMS Microbiol. Ecol. 44, 203-215. 
Painter, K.J., Hillen, T., 2002. Volume-filling and quorum-sensing in models for chemosensitive movement. Canad. Appl. Math. Quart. 10, 501-543.

Parales, R.E., Ditty, J.L., Harwood, C.S., 2000. Toluene-degrading bacteria are chemotactic towards the environmental pollutants benzene, toluene, and trichloroethylene. Appl. Environ. Microbiol. 66, 4098-4104.

Park, S., Wolanin, P.M., Yuzbashyan, E.A., Lin, H., Darnton, N.C., Stock, J.B., Silberzan, P., Austin, R., 2003. Influence of topology on bacterial social interaction. Proc. Natl. Acad. Sci. USA 100, 13910-13915.

Perthame, B., 2007. Transport Equations in Biology. Frontiers in Mathematics, Birkhäuser Verlag.

Ritter, W.F., Johnson, H.P., Lovely, W.G., 1973. Diffusion of atrazine, propachlor, and diazinon in a silt loam soil. Weed. Sci. 21, 381-384.

Rosen, G., 1983. Analytical solutions for distributions of chemotactic bacteria. B. Math. Biol. 45, 837-847.

Salman, H., Zilman, A., Loverdo, C., Jeffroy, M., Libchaber, A., 2006. Solitary modes of bacterial culture in a temperature gradient. Phys. Rev. Lett. $97,118101$.

Saragosti, J., Calvez, V., Bournaveas, N., Buguin, A., Silberzan, P., Perthame, B., 2010. Mathematical description of bacterial traveling pulses. PLoS Comput. Biol. 6, e1000890.

Schofield, P., Chaplain, M., Hubbard, S., 2002. Mathematical modelling of host-parasitoid systems: effects of chemically mediated parasitoid foraging 
618

strategies on within- and between-generation spatio-temporal dynamics. J. Theor. Biol. 214, 31-47.

Turing, A.M., 1952. The chemical basis of morphogenesis. Philos. Trans. R. Soc. London Ser. B 237, 37-72.

Tyson, R., Lubkin, S.R., Murray, J.D., 1999. Model and analysis of chemotactic bacterial patterns in a liquid medium. J. Math. Biol. 38, 359-375.

Wang, M., Ford, R.M., Harvey, R.W., 2008. Coupled effect of chemotaxis and growth on microbial distributions in organic-amended aquifer sediments: observations from laboratory and field studies. Environ. Sci. Technol. 42, $3556-3562$.

Wenderoth, D.F., Rosenbrock, P., Abraham, W.R., Pieper, D.H., Höfle, M.G., 2003. Bacterial community dynamics during biostimulation and bioaugmentation experiments aiming at chlorobenzene degradation in groundwater. Microbial. Ecol. 46, 161-176.

Woodward, D.E., Tyson, R., Myerscough, M.R., Murray, J.D., Budrene, E.O., Berg, H.C., 1995. Spatio-temporal patterns generated by salmonella typhimurium. Biophys. J. 68, 2181-2189. 
635

\section{Tables}

Table 1: Parameter values for individual-based simulations, based on typical values for E. coli

\begin{tabular}{|c|c|c|c|}
\hline \multicolumn{2}{|c|}{ Parameter } & \multirow{2}{*}{$\begin{array}{l}\text { Value } \\
\qquad 0.15 \mathrm{~min}^{-1}\end{array}$} & \multirow{2}{*}{$\frac{\text { Reference }}{\text { et al. (2006) }}$} \\
\hline$\mu$ & bacterial growth & & \\
\hline$d$ & bacterial death & $0.059 \min ^{-1}$ & Keymer et al. (2006) \\
\hline$K$ & carrying capacity of microhabitats & 10000 cell & Keymer et al. (2006) \\
\hline$\lambda$ & substrate inflow & $0.004 \min ^{-1}$ & Keymer et al. (2006) \\
\hline$\varepsilon$ & conversion efficiency & 0.15 & Keymer et al. (2006) \\
\hline$D_{s}$ & substrate diffusion coefficient & $9 \times 10^{-6} \mathrm{~cm}^{2} / \mathrm{s}$ & Berg (1983) \\
\hline$D_{p}$ & bacterial diffusion coefficient & $5.19 \times 10^{-6} \mathrm{~cm}^{2} / \mathrm{s}$ & Berg and Turner (1990) \\
\hline$\chi_{s}$ & chemotactic sensitivity to substrate & $1.3 \times 10^{-3} \mathrm{~cm}^{2} / \mathrm{s}$ & Berg and Turner (1990) \\
\hline$\chi_{p}$ & chemotactic sensitivity to bacteria & $1.3 \times 10^{-9}-1.3 \times 10$ & ${ }^{-8} \mathrm{~cm}^{2} / \mathrm{s}$ \\
\hline$\Delta x$ & distance between microhabitats & $0.005 \mathrm{~cm}$ & Keymer et al. (2006) \\
\hline$\Delta t$ & time step size & $1 \mathrm{~s}$ & \\
\hline
\end{tabular}




\section{Figures}

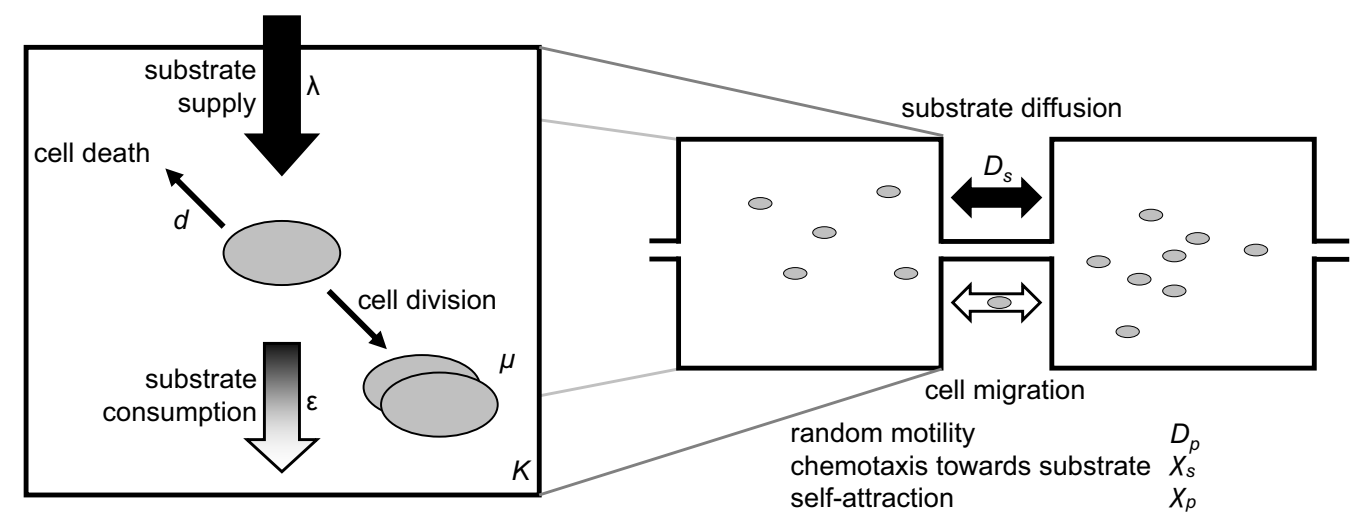

Figure 1: Key processes considered in the local (left) and spatial part (right) of the full model. Substrate supply to microhabitats is controlled by the rate parameter $\lambda$, bacterial cells consume substrate with conversion efficiency $\epsilon$ and undergo cell divisions and cell death controlled by the rate parameters $\mu$ and $d$, respectively. Microhabitats are treated as well-stirred reactors that can hold a maximum of $K$ cells. In a linear arrangement, neighboring habitats are connected by corridors through which substrate diffuses and cells can migrate due three processes, controlled by parameters as indicated. 

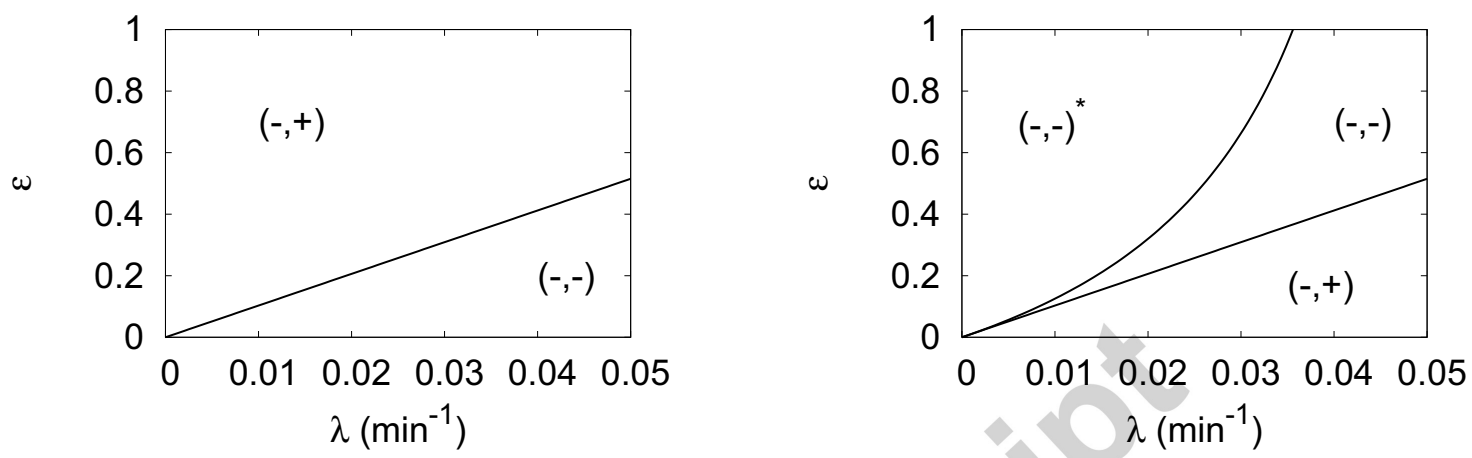

Figure 2: Stability of steady states two and three of the local model using values for parameters $\mu, d$, and $K$ as given in Table 1 . The signs of the two eigenvalues are shown for steady state two (left) and steady state three (right) in $\lambda-\varepsilon$ parameter space. Regions with saddle nodes are marked with $(-,+)$, regions with stable nodes with (-,-), and regions with stable spirals with $(-,-)^{*}$. 

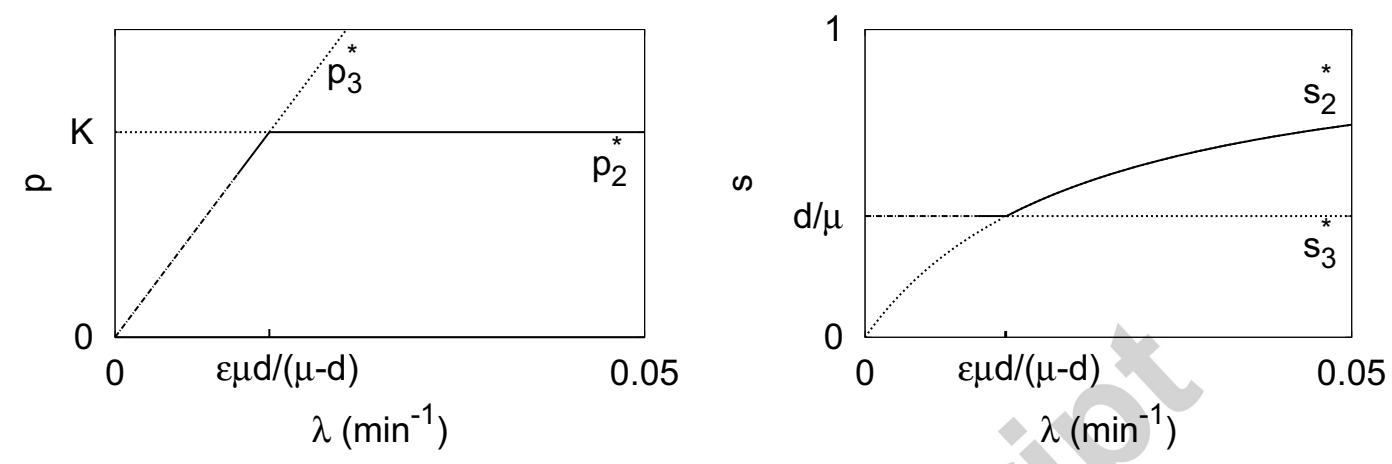

Figure 3: Bifurcation diagram for the local model using parameter values given in Table 1. Stable node as solid line, saddle point as dotted line, and stable spiral as dash-dotted line. For large $\lambda$ values, steady state two $\left(p_{2}^{*}, s_{2}^{*}\right)$ is asymptotically stable, and steady state three $\left(p_{3}^{*}, s_{2}^{*}\right)$ is unstable. When decreasing $\lambda$, stability properties are first swapped at a transcritical bifurcation, and a further bifurcation ends in a stable spiral for steady state three. 

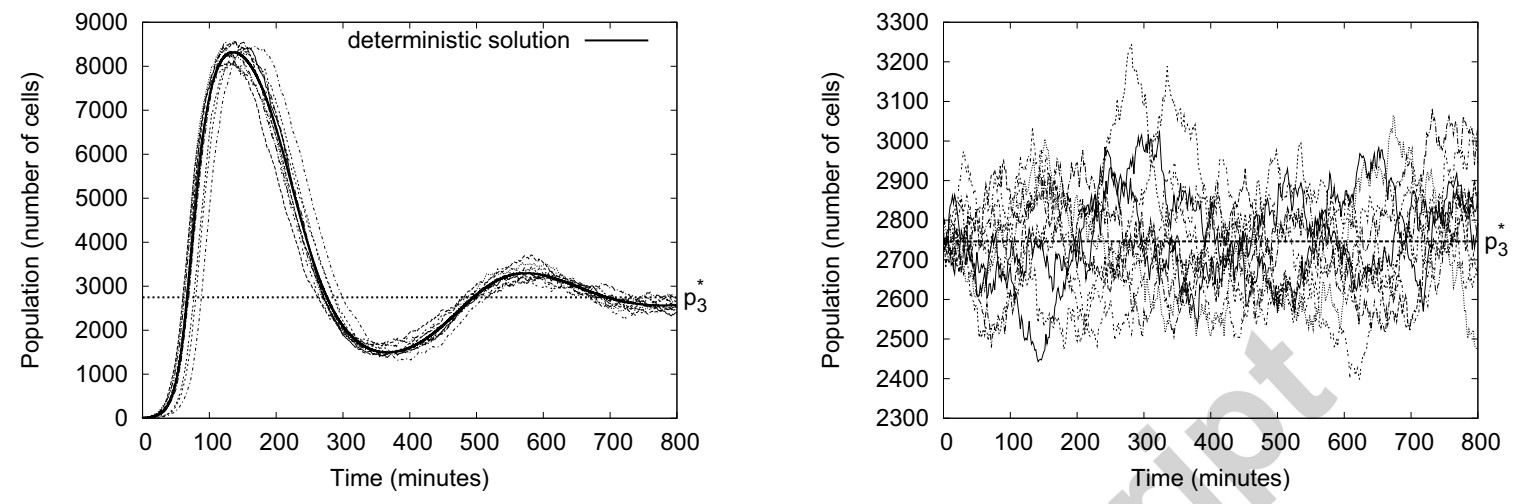

Figure 4: Growth dynamics in the local model. The deterministic solution of the local growth model is compared to ten independent individual-based model runs. The system is initialized either with ten bacterial cells and maximal substrate concentration (left), or at steady state three $\left(p_{3}^{*}=2747, s_{3}^{*}=0.394\right)$ (right). 

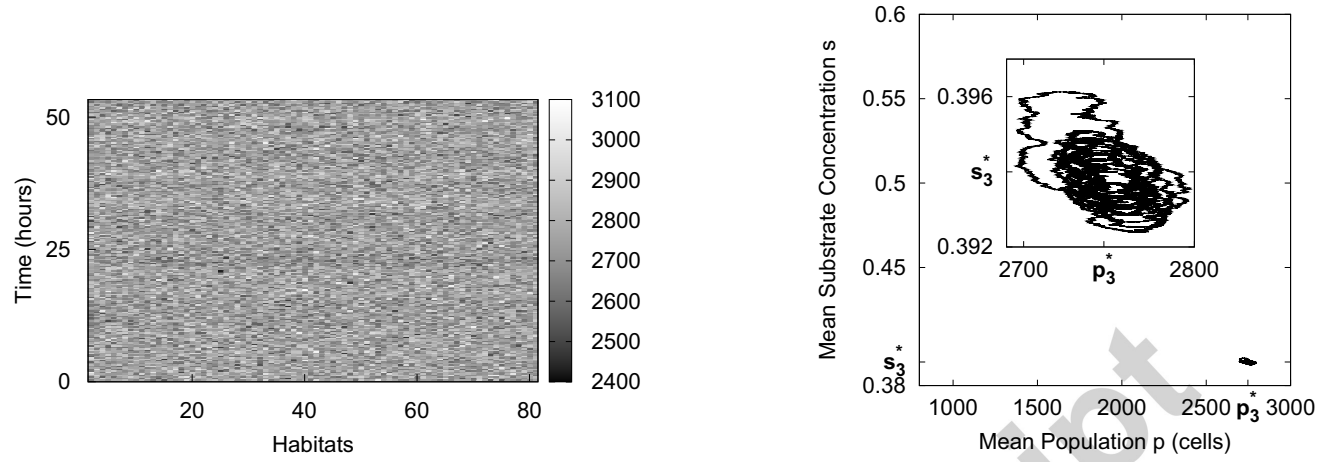

Figure 5: Homogeneous bacterial distribution for simulation runs with $\chi_{p}<\chi_{p}^{*}$. Spatio-temporal bacterial distribution (left), and mean behavior of the whole array over time (right) for a typical simulation run with $\chi_{p}=1.3 \times 10^{-9} \mathrm{~cm}^{2} / \mathrm{s}$. The mean values oscillate around steady state three of the local model (right, inset). The system is homogeneously initialized in steady state three, and all other parameters are given in Table 1. 
Pattern Type 1

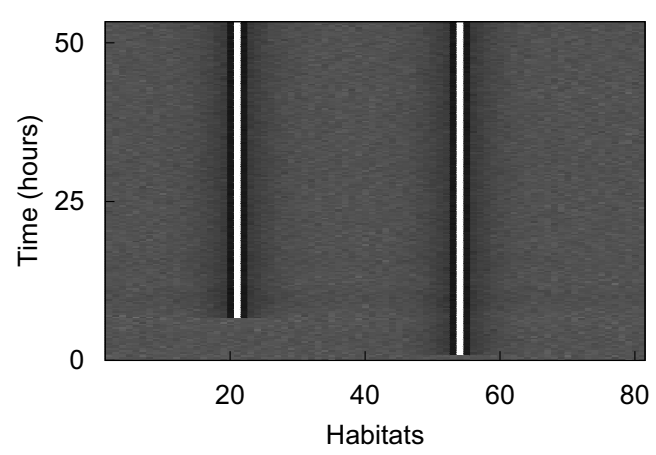

Pattern Type 3

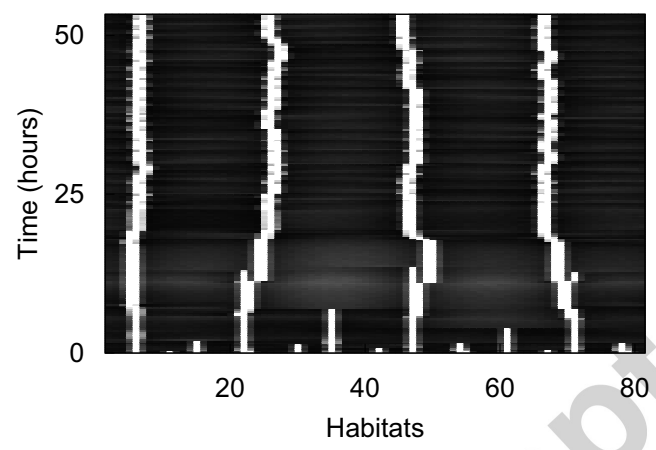

Pattern Type 2

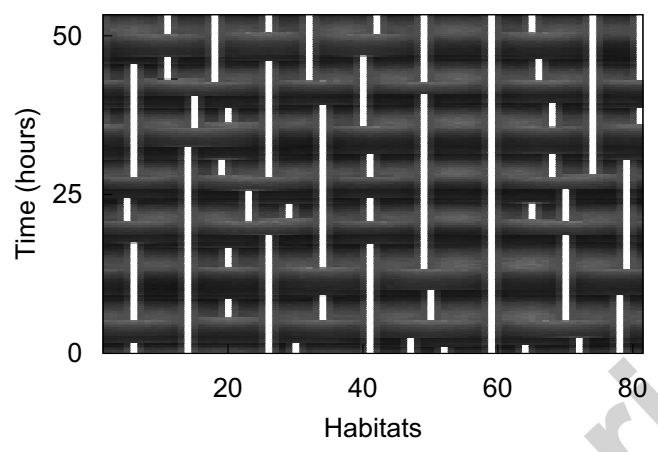

Pattern Type 4

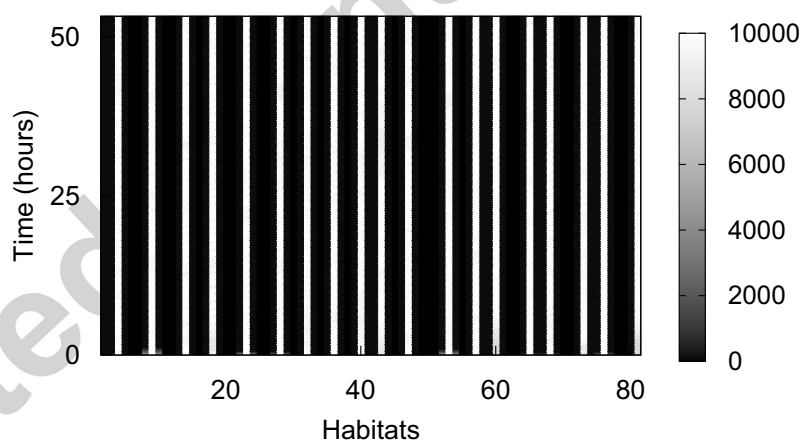

Figure 6: Spatio-temporal bacterial distribution patterns for individual simulation runs representing the four types of pattern as the pattern formation threshold $\chi_{p}^{*}$ is exceeded. Pattern formation commences shortly before $\chi_{p}^{*}=1.89 \times 10^{-9} \mathrm{~cm}^{2} / \mathrm{s}$ is reached and leads to few static hot spots in Pattern Type $1\left(\chi_{p}=1.71 \times 10^{-9} \mathrm{~cm}^{2} / \mathrm{s}\right)$, transient hot spots in Pattern Type $2\left(\chi_{p}=1.91 \times 10^{-9} \mathrm{~cm}^{2} / \mathrm{s}\right)$, moving hot spots in Pattern Type $3\left(\chi_{p}=\right.$ $\left.2.07 \times 10^{-9} \mathrm{~cm}^{2} / \mathrm{s}\right)$, and dense static hot spots in Pattern Type $4\left(\chi_{p}=1.3 \times 10^{-8} \mathrm{~cm}^{2} / \mathrm{s}\right)$. The system is homogeneously initialized in steady state three, and all other parameters are given in Table 1. 
Pattern Type 1

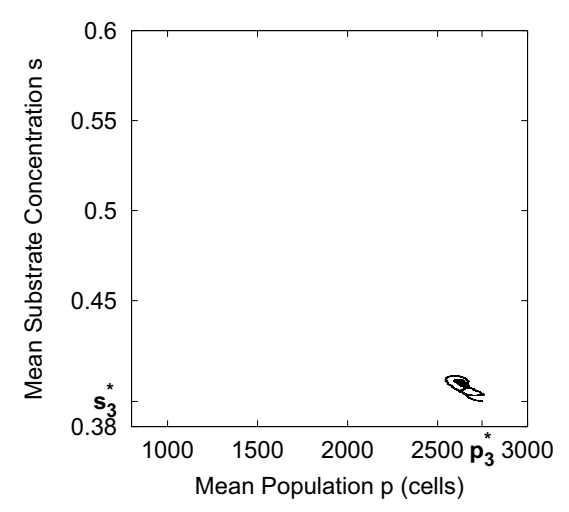

Pattern Type 3

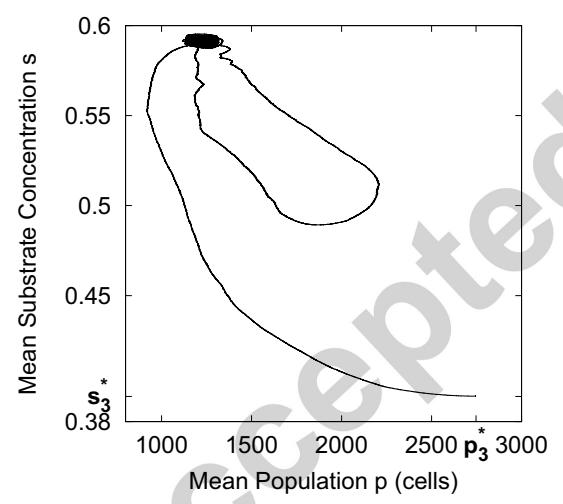

Pattern Type 2

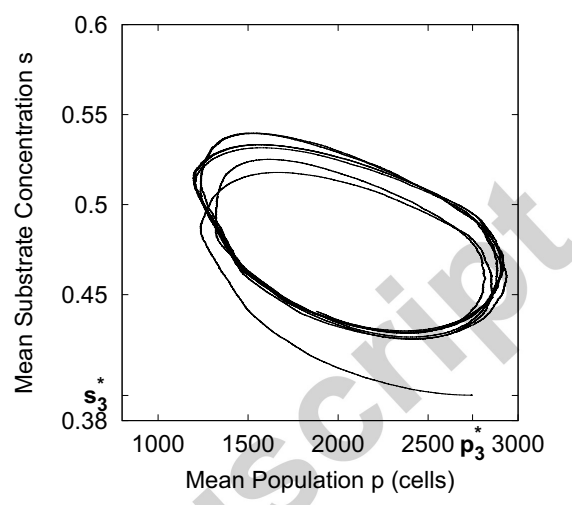

Pattern Type 4

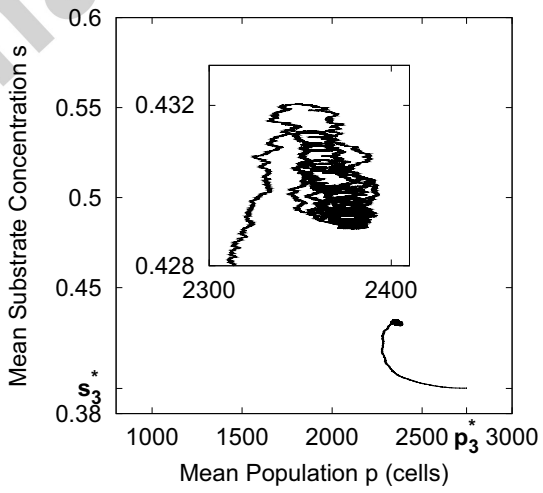

Figure 7: Temporal evolution of mean behavior over space of bacterial population and substrate concentration. Shown data are individual simulation runs representing the four types of pattern: few static hot spots (Pattern Type 1), transient hot spots (Pattern Type 2), moving hot spots (Pattern Type 3), and dense static hot spots (Pattern Type 4). The system is initialized in steady state three of the local model as indicated by $p_{3}^{*}$ and $s_{3}^{*}$. 


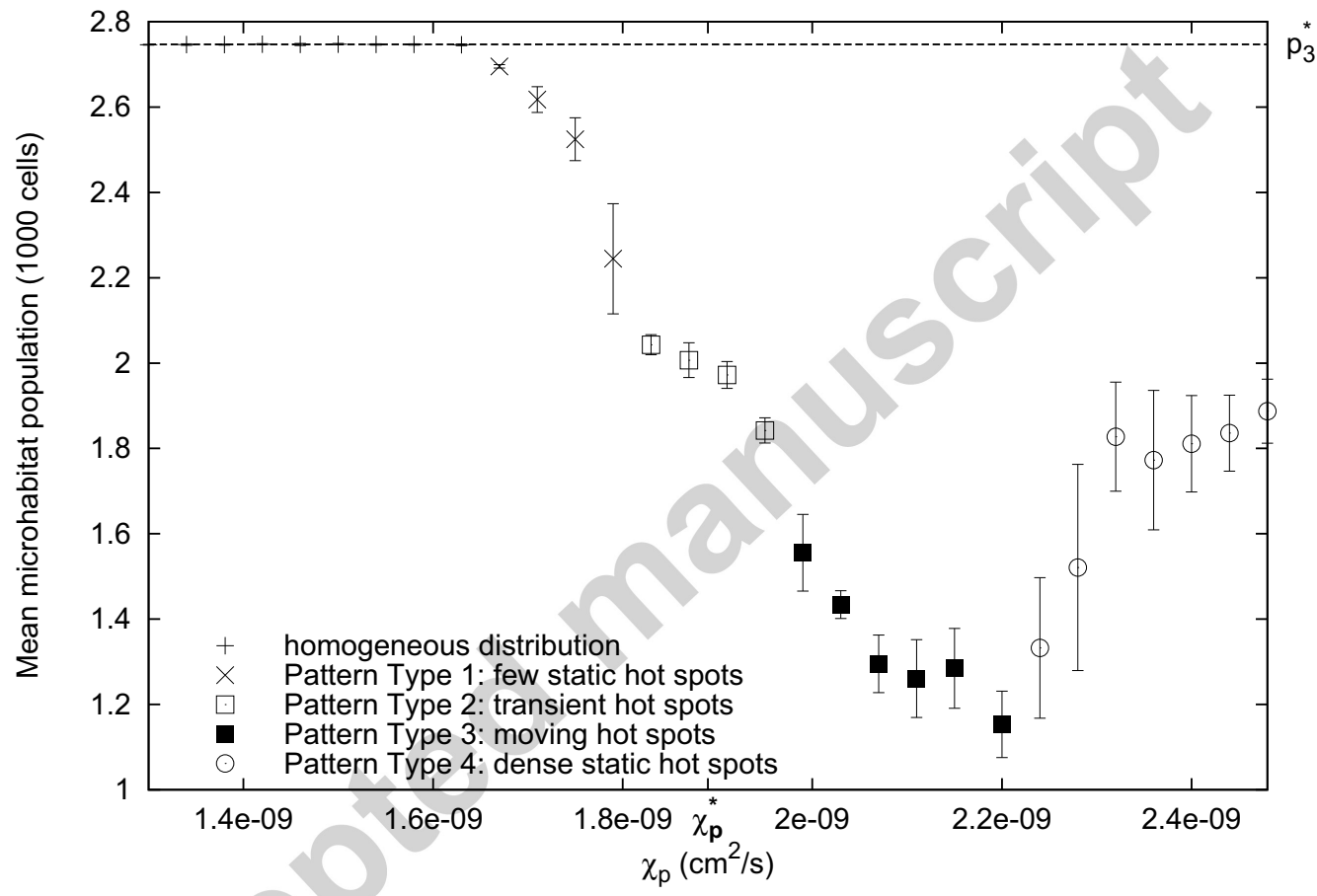

Figure 8: Changes of mean microhabitat population size when crossing the pattern formation threshold $\chi_{p}^{*}$. Symbols and error bars represent averages and standard deviations over ten independent simulation runs. Pattern formation commences shortly before $\chi_{p}^{*}$ is reached and consists of four types of distinct spatio-temporal population patterns. 

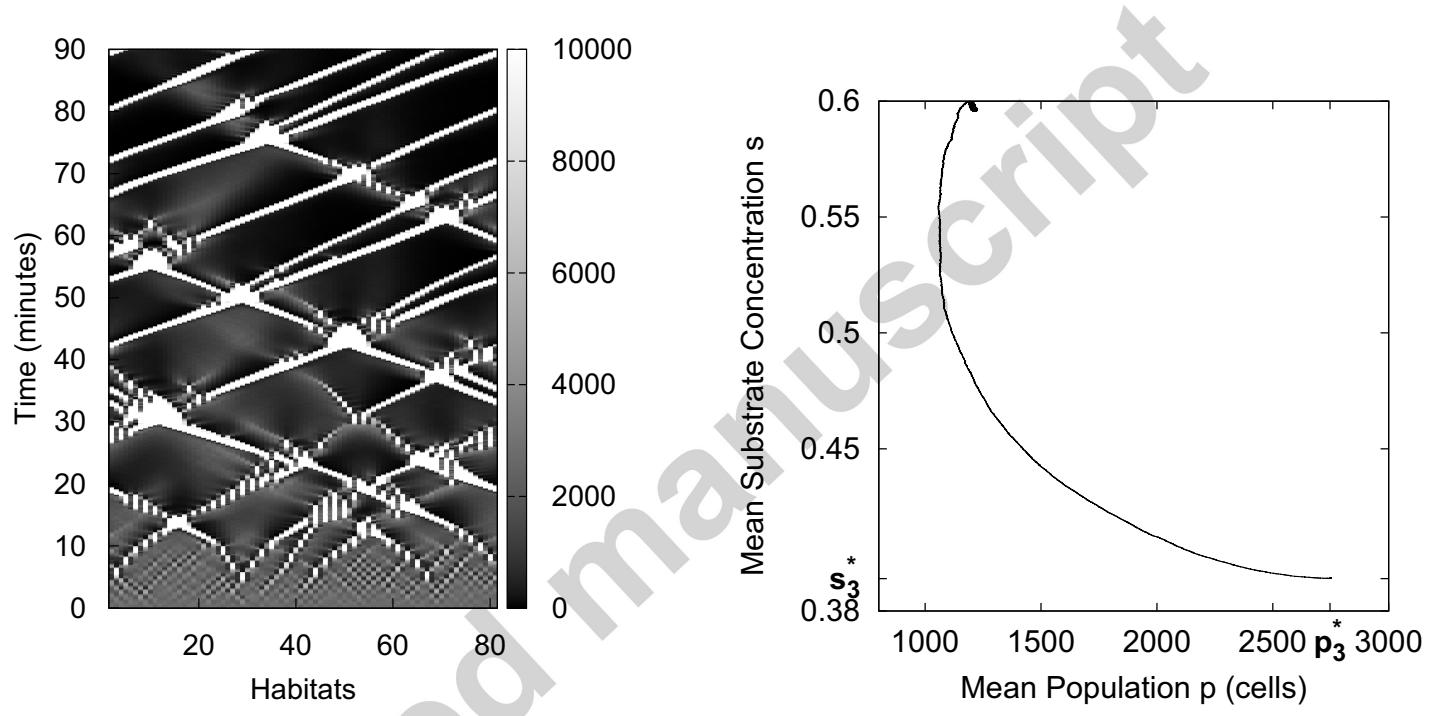

Figure 9: Spatio-temporal bacterial distribution (left), and mean behavior of bacterial population and substrate concentration over time (right) for a substrate diffusion coefficient 50 times lower than in other simulation runs $\left(D_{s}=1.8 \times 10^{-7} \mathrm{~cm}^{2} / \mathrm{s}\right)$. The chemotactic sensitivity towards chemoattractant is set to a value above the pattern formation threshold $\left(\chi_{p}=1.99 \times 10^{-9} \mathrm{~cm}^{2} / \mathrm{s}\right)$. The system is homogeneously initialized in steady state three, and all other parameters are given in Table 1. 

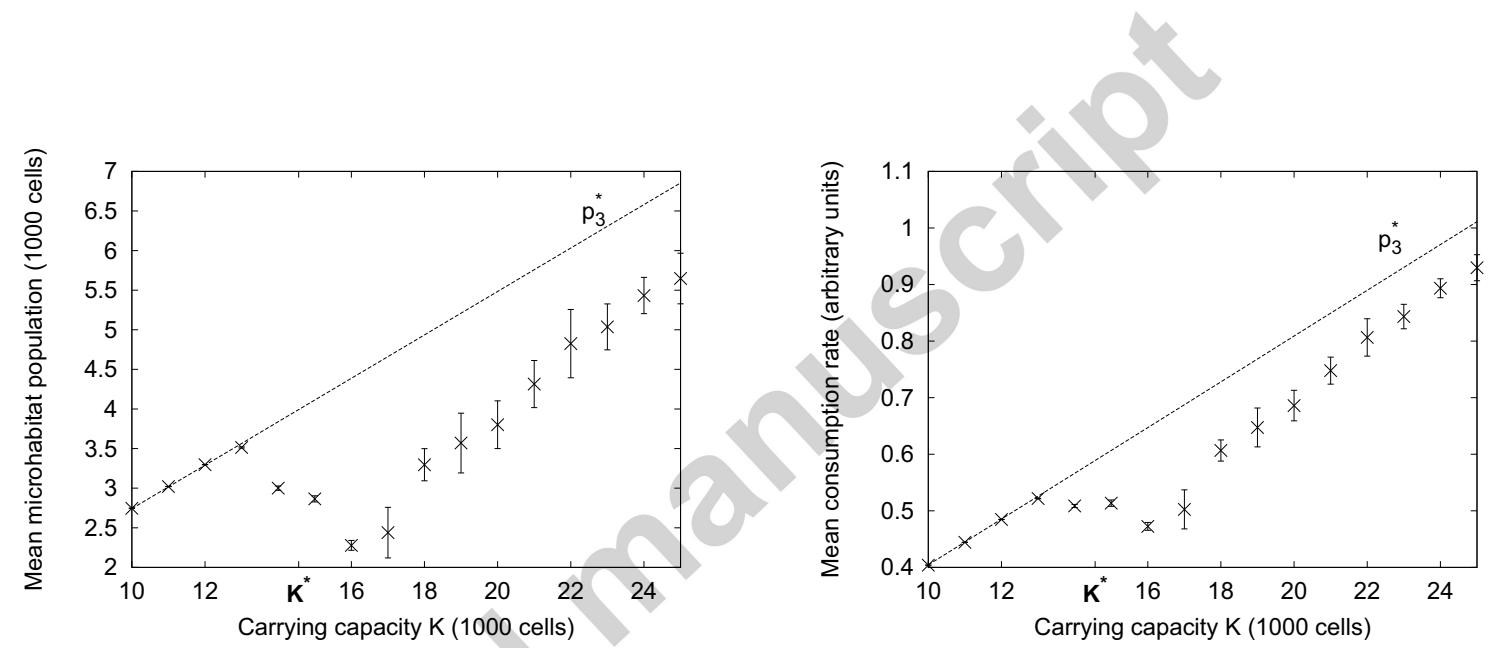

Figure 10: Response to biostimulation (increasing $K$ values). After an initially linear response of population density (left) and substrate consumption rate (right) to biostimulation matching steady state three of the local model $\left(p_{3}^{*}\right)$, biodegradation performance transiently decreases as pattern formation commences close to $K^{*}=14560$ bacterial cells. Mean over ten independent simulation runs, error bars indicate standard deviation. 
* pattern formation depends on bacterial physiology and environmental parameters

* inhomogeneous bacterial distributions lead to reduced substrate utilization

* onset of pattern formation leads to nonlinear response during biostimulation 